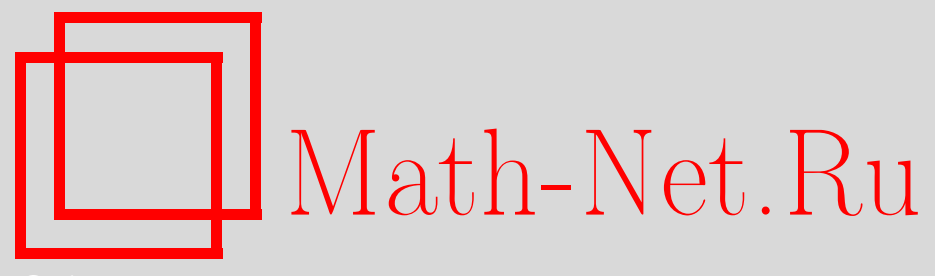

Б. А. Погорелов, М. А. Пудовкина, Орбитальные производные над кольцом вычетов. Часть II. Вероятностно-комбинаторные свойства, Матем. вопр. криптогр., 2015, том 6, выпуск 1,117133

DOI: https://doi.org/10.4213/mvk154

Использование Общероссийского математического портала Math-Net.Ru подразумевает, что вы прочитали и согласны с пользовательским соглашением http://www.mathnet.ru/rus/agreement

Параметры загрузки:

IP : 54.224 .135 .184

26 апреля 2023 г., 15:09:07

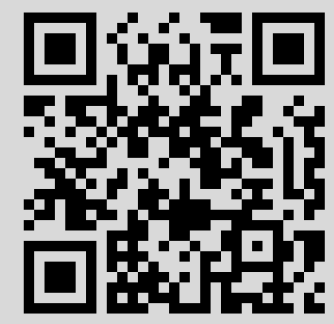




\title{
Орбитальные производные над кольцом вычетов. Часть II. Вероятностно- комбинаторные свойства
}

\author{
Б. А. Погорелов ${ }^{1}$, М. А. Пудовкина ${ }^{2}$ \\ ${ }^{1}$ Академия криптографии Российской Федерации, Москва \\ ${ }^{2}$ Национальный исследовательский ядерный университет (МИФИ), Москва
}

Получено 22.IV.2014

Рассматриваются вероятностные и комбинаторные свойства орбитальной производной $r$-го порядка над кольцом вычетов. Приведены значения орбитальной производной $r$-го порядка для множеств отображений:

1) используемых в некоторых алгоритмах шифрования;

2) являющихся сплетением симметрических групп.

Ключевые слова: орбитальная производная, сплетение групп подстановок, криптографические свойства

Orbital derivatives on the residue ring. Part II. Probabilistic and combinatorial properties
B. A. Pogorelov ${ }^{1}$, M. A. Pudovkina ${ }^{2}$
${ }^{1}$ Academy of Cryptography of the Russian Federation, Moscow
${ }^{2}$ National Nuclear Research University, Moscow

\begin{abstract}
Probabilistic and combinatorial properties of higher order orbital derivatives on the residue ring are considered. In particular, higher order orbital derivatives are given for two classes of mappings. The first class is used in block ciphers; the second class is a subset of the wreath product of permutation groups.
\end{abstract}

Keywords: orbital derivative, wreath product of permutation groups, cryptographic properties

Citation: Mathematical Aspects of Cryptography, 2015, vol. 6, no. 1, pp. 117-133 (Russian) 


\section{1. Введение}

В дискретной математике и криптографии для отображения $f: X \rightarrow X^{\prime}$ на абелевых группах $(X,+),\left(X^{\prime},+\right)$ производная по направлению $\delta \in X$ чаще всего задается в виде разности $\Delta_{\delta} f(\alpha)=f(\alpha+\delta)-f(\alpha)$ (см., например, [1]). Случай $\delta=1, X=\mathbb{Z}_{2^{n}}, X^{\prime}=\mathbb{Z}_{2^{m}}$ имеет место в исчислении конечных разностей (см. $[2,3,4,5])$. При подобных подходах, на наш взгляд, не в полной мере учитываются алгебраические и подстановочные свойства групп, хотя эти свойства могут влиять на криптографические параметры отображений [6]. Более того, возможно существование направлений, относительно которых последовательность производных является периодической, что не позволяет вводить параметры отображений типа алгебраической степени.

В [7] для более глубокого учета алгебраических свойств абелевых групп $(X,+),\left(X^{\prime},+\right)$, где $X^{\prime}-$ подгруппа группы $X$, предложено определение орбитальной производной, учитывающее строение смежных классов по подгруппам группы $X$ : значение производной в точке $x$ определяется суммированием значений по смежному классу подгруппы, содержащему эту точку. В частности, для отображений, заданных на кольце вычетов $\mathbb{Z}_{2^{n}}$, определение учитывает строение орбит подгрупп аддитивной группы $\mathbb{Z}_{2^{n}}^{+}$ кольца вычетов $\mathbb{Z}_{2^{n}}$ в ее регулярном представлении. Группа $\mathbb{Z}_{2^{n}}^{+}$состоит из сдвигов $\varphi_{\alpha}: x \mapsto x+\alpha\left(\bmod 2^{n}\right), \alpha \in \mathbb{Z}_{2^{n}}$. Рассматривались основные свойства оператора $\Delta_{+}^{(r)}$ взятия орбитальной производной $r$-го порядка, которому соответствует подгруппа $\left\langle\varphi_{2^{n-r}}\right\rangle$ порядка $2^{r}$, а значение орбитальной производной $\Delta_{+}^{(r)} f(\beta)$ определяется суммированием значений отображения $f$ на смежном классе $\beta+\left\langle 2^{n-r}\right\rangle\left(\bmod 2^{n}\right)$ (орбите группы $\left\langle\varphi_{2^{n-r}}\right\rangle$, содержащей точку $\beta$ ).

В настоящей работе продолжается изучение оператора $\Delta_{+}^{(r)}$. В его терминах рассматриваются вероятностные и комбинаторные свойства отображений. Приведены значения орбитальной производной $r$-го порядка для множеств отображений:

1) используемых в некоторых алгоритмах шифрования;

2) являющихся сплетением симметрических групп.

Показано, что в терминах орбитальной производной можно разбивать ключевое множество на множества «эквивалентных» ключей. В частности, для ключей шифрования, принадлежащих одному множеству «эквивалентных» 
ключей, одинаковы значения орбитальной производной функции зашифрования на этих ключах. Это создает возможность для применения метода криптоанализа, основанного на эквивалентных ключах. Также рассмотрен $l$-раундовый XSL-алгоритм блочного шифрования с независимыми раундовыми ключами, для которого получены соотношения, связывающие орбитальные производные $t$-раундовой функции зашифрования и шифртексты, $t<l$.

\section{2. Обозначения и предварительные сведения}

Пусть $\mathbb{N}$ - множество всех натуральных чисел, $\mathbb{N}_{0}=\mathbb{N} U\{0\} ; \mathbb{Z}-$ кольцо целых чисел; $Z_{m}=\{0, \ldots, m-1\} \subset \mathbb{Z} ; \mathbb{Z}_{2^{n}}$ - кольцо вычетов по модулю $2^{n}$, элементы которого обозначаются числами $0, \ldots, 2^{n}-1$; при $d<n$ для $\alpha \in Z_{2^{n}}$ через $\alpha_{(d)}$ обозначаем такой элемент $\alpha_{(d)} \in Z_{2^{d}}$, что $\alpha_{(d)} \equiv \alpha \bmod 2^{d}$, при этом $\alpha_{(0)}=0, x \mapsto x_{(d)}-$ соответствующее отображение; $A_{(d)}=\left\{\alpha_{(d)} \mid \alpha \in A\right\}$ для $A \subset \mathbb{Z} ; V_{n}$ - пространство $n$-мерных векторов над полем $G F(2)$, т. е. $V_{n}=V_{n}(2) ; \oplus-$ операция покоординатного сложения векторов в пространстве $V_{n} ; S(X)$ - множество всех подстановок на множестве $X$; $X^{\times}=X \backslash\{0\}$, если 0 - нейтральный элемент относительно заданной на $X$ операции; $\left\langle\beta_{1}, \ldots, \beta_{t}\right\rangle$ - подгруппа группы $X$ с некоторой бинарной опера-

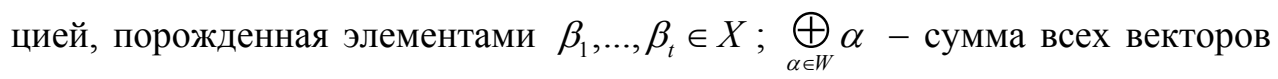
из множества $W \subseteq V_{n} ;\left(\alpha_{1}, \ldots, \alpha_{t}\right) \equiv_{2^{n}}\left(\beta_{1}, \ldots, \beta_{t}\right)$ - покоординатное равенство в кольце $\mathbb{Z}_{2^{n}}, \quad t \geq 1 ; \quad P\left(\mathbb{Z}_{2^{n}}\right)$ - множество всех отображений $\mathbb{Z}_{2^{n}} \rightarrow \mathbb{Z}_{2^{n}}$; $\alpha^{g}=\alpha g=g(\alpha)$ - образ элемента $\alpha \in X$ при действии на него подстановкой $g \in S(X) ; \alpha^{G}=\left\{\alpha^{g} \mid g \in G\right\}, G \leq S(X) ; A^{*} B=\left\{\alpha^{*} \beta \mid(\alpha, \beta) \in A \times B\right\}$ для бинарной операции «*» группы $X$ и $A, B \subset X$; группы $\mathbb{Z}_{2^{n}}^{+}$и $V_{n}^{+}-$аддитивные группы кольца $\mathbb{Z}_{2^{n}}$ и векторного пространства $V_{n} ; a(x) \cdot b(x) \equiv_{2^{n}} c(x)$ есть сокращенная запись для $a(x) \cdot b(x) \equiv c(x)\left(\bmod 2^{n}\right)$ для всех $x \in \mathbb{Z}_{2^{n}}$, где $a(x), b(x)$ - произвольные отображения, «·»- бинарная операция над числами в кольце $\mathbb{Z}$, представляющими соответствующие классы вычетов; запись $a *_{2^{n}} A$ означает, что элемент $a_{(n)}$ и множество $A_{(n)}$ находятся в отношении $* \in\{\in, \notin\}, a \in \mathbb{Z}, A \subset \mathbb{Z}$; запись $B *_{2^{n}} C$ означает, что множества $B_{(n)}$ и $C_{(n)}$ находятся в отношении $* \in\{\subseteq, \not \subset,=\}, B, C \subset \mathbb{Z} ; \mathbb{Z}_{2^{m}}^{c}-c$-я декартова 
степень множества $\mathbb{Z}_{2^{m}} ; x \in_{U} X$ означает, что элемент $x$ случайно и равновероятно выбирается из множества $X$;

$$
U_{n, t}(\delta)=\left\{\delta+2^{t} c \mid c \in\left\{0, \ldots, 2^{n-t}-1\right\}\right\}=\delta+\left\langle 2^{t}\right\rangle\left(\bmod 2^{n}\right) \text { для } \delta \in Z_{2^{n}} .
$$

Вектор $\alpha=\left(\alpha_{n-1}, \ldots, \alpha_{1}, \alpha_{0}\right) \in V_{n}$ отождествляется с числом $\tilde{\alpha}=2^{n-1} \alpha_{n-1}+$ $+\ldots+2 \alpha_{1}+\alpha_{0}$. Тем самым на пространстве $V_{n}$ определяются операции сложения и умножения по модулю $2^{n}$, а над кольцом $\mathbb{Z}_{2^{n}}-$ операция $\oplus$ сложения векторов. Эти операции будем обозначать теми же символами, что и соответствующие операции над числами или векторами. Из контекста всегда будет ясно, о каких операциях идет речь.

Всюду ниже предполагается, что $n \geq 2$.

В работе [7] даны следующие определения.

Определение 1. Орбитальной производной $r$-го порядка отображения $f: \mathbb{Z}_{2^{n}} \rightarrow \mathbb{Z}_{2^{m}}, n \in \mathbb{N}, m, r \in\{1, \ldots, n\}$, называется оператор $\Delta_{+}^{(r)}$, задаваемый формулой

$$
\Delta_{+}^{(r)} f(x) \equiv_{2^{m}} \sum_{\beta \in U_{n, n-r}(x)} f(\beta) .
$$

Наименьшее число $r \in \mathbb{N}$, при котором для отображения $f: \mathbb{Z}_{2^{n}} \rightarrow \mathbb{Z}_{2^{m}}, \quad m \leq n$, существует элемент $\lambda \in \mathbb{Z}_{2^{m}}$, удовлетворяющий равенству $\Delta_{+}^{(r)} f(x) \equiv_{2^{m}} \lambda$, называется $\Delta_{+}$-степенью отображения $f$ и обозначается $d_{+}(f)$. Очевидно, что $d_{+}(f) \leq n$.

Для функции $f: V_{n} \rightarrow\{0,1\}$ производная по направлению $\beta_{1} \in V_{n}$ задается оператором $\Delta_{\oplus, \beta_{1}}$ :

$$
\Delta_{\oplus, \beta_{1}} f(x)=\Delta_{\oplus, \beta_{1}}^{(1)} f(x)=f\left(x \oplus \beta_{1}\right) \oplus f(x),
$$

а $r$-й (высшей) производной по направлению $\beta_{1}, \ldots, \beta_{r}$ из $V_{n}$ называется сумма $\Delta_{\oplus, \beta_{r}, \ldots, \beta_{1}}^{(r)} f(x)=\Delta_{\oplus, \beta_{r}}^{(1)} \ldots \Delta_{\oplus, \beta_{2}}^{(1)}\left(\Delta_{\oplus, \beta_{1}}^{(1)} f(x)\right)$.

\section{3. Орбитальные производные для некоторых классов отображений}

Силовская 2-подгруппа группы $S_{2^{n}}$ изоморфна $n$-кратному сплетению групп $S_{2} 2 S_{2} 2 \ldots 2 S_{2}$ и содержит группы $V_{n}^{+}$и $\mathbb{Z}_{2^{n}}^{+}$. В терминах операции сплетения описываются отображения, обладающие линейными структура- 
ми [8]. Найдем орбитальную производную $r$-го порядка для отображений из сплетения групп подстановок.

Пусть $W=\left\{W_{0}, \ldots, W_{p-1}\right\}$ - нетривиальное разбиение множества $X$ на $p$ подмножеств мощности $w,|X|=p \cdot w$, т. е. $W \notin\{\{X\},\{\{\alpha\} \mid \alpha \in X\}\}$; $W_{w, p}$ - множество всех таких разбиений с параметрами $p, w$; $I G_{W}=\left(S_{w} \prec S_{p}, W\right) \quad$ - максимальная группа подстановок на $X=W_{0} \cup \ldots \cup W_{p-1}$, сохраняющая разбиение $W \in W_{w, p}$. Эта группа называется сплетением группы подстановок $S_{w}$ группой $S_{p}$.

Лемма 3.1. Пусть $n \geq 2, \quad r \in\{1, \ldots, n-1\}, \quad d \in \mathbb{N}_{0}, \quad f \in I G_{W}$, $W=\left\{W_{0}, \ldots, W_{2^{n-r}-1}\right\}, \quad W_{j}=\left\{\beta \in \mathbb{Z}_{2^{n}} \mid \beta \equiv_{2^{n-r}} j\right\}, \quad\left(W_{j}\right)^{f}=W_{j^{b_{f}}}$ для некоторой подстановки $b_{f} \in S\left(Z_{2^{n-r}}\right)$. Тогда

$$
\Delta_{+}^{(r+d)} f(x) \equiv_{2^{n}} \begin{cases}2^{r}\left(x_{(n-r)}\right)^{b_{f}}+2^{n-1}, & \text { если } d=0, \\ 2^{r} \Delta_{+}^{(d)}\left(x_{(n-r)}\right)^{b_{f}}, & \text { если } d>0 .\end{cases}
$$

Доказательство. Если $d=0$, то

$$
\begin{aligned}
\Delta_{+}^{(r)} f(x) & \equiv \sum_{2^{n}} \sum_{\gamma \in Z_{2^{r}}} f\left(2^{n-r} \gamma+x_{(n-r)}\right) \equiv \sum_{2^{n}} \sum_{\beta \in W_{\left(x_{(n-r)}\right)^{b_{f}}}} \beta=2^{r}\left(x_{(n-r)}\right)^{b_{f}}+2^{n-r} \sum_{\gamma \in Z_{2^{r}}} \gamma \equiv_{2^{n}} \\
& \equiv_{2^{n}} 2^{r}\left(x_{(n-r)}\right)^{b_{f}}+2^{n-1}\left(2^{r}-1\right) \equiv_{2^{n}} 2^{r}\left(x_{(n-r)}\right)^{b_{f}}+2^{n-1} .
\end{aligned}
$$

При $d>0$ из леммы 3.1 работы [7] получаем

$$
\begin{aligned}
& \Delta_{+}^{(r+d)} f(x) \equiv \sum_{2^{n}} \sum_{\beta \in Z_{2^{d}}} \Delta_{+}^{(r)} f\left(x+2^{n-r-d} \beta\right) \equiv \\
& \quad \equiv 2_{2^{n}} 2^{r} \sum_{\beta \in Z_{2^{d}}}\left(\left(x+2^{n-r-d} \beta\right)_{(n-r)}\right)^{b_{f}}+\sum_{\beta \in Z_{2^{d}}} 2^{n-1} \equiv \sum_{2^{n}} 2^{r} \Delta_{+}^{(d)}\left(x_{(n-r)}\right)^{b_{f}} .
\end{aligned}
$$

Лемма доказана.

Согласно [7] для $q \in \mathbb{N}$ отображение $f \in P\left(\mathbb{Z}_{2^{n}}\right)$ называется $(r, q)$-отображением, если $2^{r} q=\left|\left\{\alpha \in \mathbb{Z}_{2^{n}} \mid \Delta_{+}^{(r)} f(\alpha) \equiv_{2^{n}} \beta\right\}\right| \quad$ для $\quad$ всех $\beta \in\left\{\Delta_{+}^{(r)} f(x) \mid x \in \mathbb{Z}_{2^{n}}\right\}$. Пусть $\quad \mathrm{B}_{r, q}^{(s)} \quad-$ множество всех биективных $(r, q)$ - отображений над $\mathbb{Z}_{2^{n}}$. Покажем, что всегда найдется такое разбиение $W$, что $\mathrm{B}_{t, 2^{n-t}}^{(s)} \cap I G_{W} \neq \varnothing$ для $t \geq 2$. 
Следствие 3.2. Если $n \geq 2, \quad r \in\{1, \ldots, n-1\}, \quad W_{j}=\left\{\beta \in \mathbb{Z}_{2^{n}} \mid \beta \equiv_{2^{n-r}} j\right\}$, $W=\left\{W_{0}, \ldots, W_{2^{n-r}-1}\right\}, t \in\{r+1, \ldots, n\}$, mo $\mathrm{B}_{t, 2^{n-t}}^{(s)} \cap I G_{W} \neq \varnothing$.

Доказательство. Положим $d=t-r$. Существует такое отображение $f \in I G_{W}$, что $\left(W_{j}\right)^{f}=W_{j^{b_{f}}}$ для некоторой подстановки $b_{f} \in \mathrm{B}_{d, 2^{n-r-d}}^{(s)}$. Так как $\Delta_{+}^{(d)} b_{f}(\beta) \equiv_{2^{n-r}} \gamma$ для некоторого $\gamma \in Z_{2^{n-r}}$ и всех $\beta \in Z_{2^{n-r}}$, то из леммы 3.1 следует, что $\Delta_{+}^{(r+d)} f(x) \equiv{ }_{2^{n}} 2^{r} \gamma$. Значит, $f \in \mathrm{B}_{t, 2^{n-t}}^{(s)} \cap I G_{W}$. Следствие доказано.

В ряде алгоритмов блочного шифрования используется линейное отображение $g$, осуществляющее перестановку координат $n$-мерного вектора. Для таких отображений укажем значение $t$-й орбитальной производной и $\Delta_{+}$-степень $d_{+}(g)$.

Лемма 3.3. Пусть $n \geq 2, s \in S(\{0, \ldots, n-1\}), t \in\{1, \ldots, n\}$, подстановка $g_{s} \in S\left(\mathbb{Z}_{2^{n}}\right)$ задана условием $g_{s}: x \mapsto \sum_{i=0}^{n-1} 2^{i} x_{i^{s}}\left(\bmod 2^{n}\right)$, где $x=\left(x_{n-1}, \ldots, x_{0}\right) \in$ $\in\{0,1\}^{n}$.

Тогда $d_{+}(g)=n u$

$$
\Delta_{+}^{(t)} g_{s}(x) \equiv 2_{2^{n}} 2^{t} g_{s}(x)+2^{t} \sum_{j=0}^{t-1} 2^{(n-1-j)^{s}} .
$$

Доказательство. Равенство (3.1) следует из сравнений

$$
\begin{aligned}
& \Delta_{+}^{(t)} g_{s}(x) \equiv \sum_{2^{n}} \sum_{\left(u_{t-1}, \ldots, u_{0}\right) \in Z_{2}^{t}}\left(\sum_{i=0}^{n-1} 2^{i} x_{i^{s}}+\sum_{j=0}^{t-1} 2^{(n-1-j)^{s}} u_{j}\right) \equiv_{2^{n}} \\
& \equiv 2_{2^{t}} g_{s}(x)+\sum_{\left(u_{t-1}, \ldots, u_{0}\right) \in Z_{2}^{t}} \sum_{j=0}^{t-1} 2^{(n-1-j)^{s}} u_{j} \equiv 2_{2^{n}} 2^{t} g_{s}(x)+2^{t} \sum_{j=0}^{t-1} 2^{(n-1-j)^{s}} .
\end{aligned}
$$

Так как

$$
\begin{gathered}
\Delta_{+}^{(n-1)} g_{s}(0) \equiv_{2^{n}} 2^{n-1} \sum_{j=0}^{t-1} 2^{(n-1-j)^{s}}, \\
\Delta_{+}^{(n-1)} g_{s}\left(2^{(0)^{-1}}\right) \equiv_{2^{n}} 2^{n-1}+2^{n-1} \sum_{j=0}^{t-1} 2^{(n-1-j)^{s}},
\end{gathered}
$$

то $\Delta_{+}^{(n-1)} g_{s}(0) \nexists_{2^{n}} \Delta_{+}^{(n-1)} g_{s}\left(2^{(0)^{s^{-1}}}\right)$. Значит, $d_{+}\left(g_{s}\right)=n$. Лемма доказана.

Из леммы 3.3 следует, что для подстановки $g \in S\left(V_{n}\right)$, осуществляющей циклический сдвиг вектора на $r$ координат (вправо или влево), 
$r \in\{1, \ldots, n-1\}, t$-я орбитальная производная находится из равенства (3.1) для $s: i \mapsto i+r(\bmod n)$ или для $s: i \mapsto i-r(\bmod n)$. Кроме того, у всех таких подстановок, включая тождественную, $\Delta_{+}$-степень равна $n$ и является наибольшей.

Заметим, что $\Delta_{+}$-степень отображения задает иную иерархию среди подстановок по сравнению с рассмотренной в [9]. Это обусловлено тем, что $\Delta_{+}$-степень отображения определяется системами импримитивности аддитивной группы $\mathbb{Z}_{2^{+}}^{+}$. Примером подстановки с наименьшей $\Delta_{+}$-степенью является подстановка $g \in S\left(\mathbb{Z}_{2^{n}}\right)$, заданная условием

$$
g: x \mapsto \begin{cases}x, & \text { если } x<2^{n-1}, \\ 2^{n-1}-1-x\left(\bmod 2^{n}\right), & \text { если } x \in\left\{2^{n-1}, 2^{n-1}+1, \ldots, 2^{n}-1\right\},\end{cases}
$$

для нее $d_{+}(g)=1$.

Найдем $t$-ю орбитальную производную и $\Delta_{+}$-степень отображения, используемого в алгоритме блочного шифрования SAFER.

Пусть $m, c \in \mathbb{N}, n=m \cdot c, \psi_{c}$ - естественная биекция $\mathbb{Z}_{2^{m}}^{c}$ в $\mathbb{Z}_{2^{n}}$, где $\psi_{c}:\left(x_{c-1}, \ldots, x_{0}\right) \mapsto \sum_{i=0}^{c-1} 2^{m \cdot i} x_{i}$. Подстановке $g \in S\left(\mathbb{Z}_{2^{m}}^{c}\right)$ поставим в соответствие подстановку $g^{[c]} \in S\left(\mathbb{Z}_{2^{n}}\right)$, заданную равенством

$$
g^{[c]}\left(\psi_{c}\left(x_{c-1}, \ldots, x_{0}\right)\right)=\psi_{c}\left(g\left(x_{c-1}, \ldots, x_{0}\right)\right) .
$$

Лемма 3.4. Пусть $m \in \mathbb{N}, n=2 m \geq 4, t \in\{m+1, \ldots, n\}$ и подстановка $g \in S\left(\mathbb{Z}_{2^{m}}^{2}\right)$ задана равенством

$$
\left.\left(x_{1}, x_{0}\right)^{g} \equiv_{2^{m}}\left(2 x_{1}+x_{0}, x_{1}+x_{0}\right)\right) .
$$

Tогда $d_{+}\left(g^{[2]}\right)=m u$

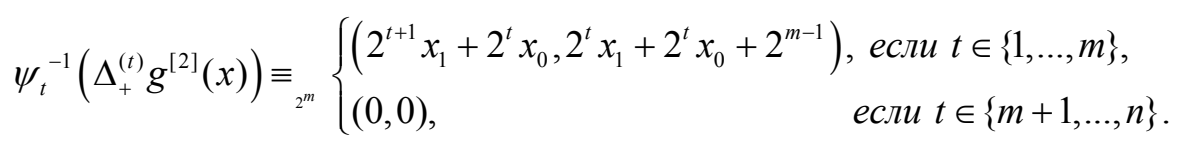

Доказательство. Если $t \in\{1, \ldots, m\}$ и $x=\psi_{2}\left(x_{1}, x_{0}\right)$, то выполняется сравнение

$$
\psi_{2}^{-1}\left(\Delta_{+}^{(t)} g^{[2]}(x)\right) \equiv \sum_{2^{m}} \sum_{c \in Z_{2^{t}}}\left(2\left(x_{1}+c \cdot 2^{m-t}\right)+x_{0}, x_{1}+c \cdot 2^{m-t}+x_{0}\right) \equiv_{2^{m}}
$$




$$
\begin{aligned}
& \equiv{ }_{2^{m}}\left(2^{t+1} x_{1}+2^{m-t+1} \cdot \sum_{c \in Z_{2^{t}}} c+2^{t} x_{0}, 2^{t} x_{1}+2^{t} x_{0}+2^{m-t} \cdot \sum_{c \in Z_{2^{t}}} c\right) \equiv_{2^{m}} \\
& \equiv{ }_{2^{m}}\left(2^{t+1} x_{1}+2^{m-t+1} \cdot 2^{t-1}\left(2^{t}-1\right)+2^{t} x_{0}, 2^{t} x_{1}+2^{t} x_{0}+2^{m-t} \cdot 2^{t-1}\left(2^{t}-1\right)\right) \equiv_{2^{m}} \\
& \equiv_{2^{m}}\left(2^{t+1} x_{1}+2^{t} x_{0}, 2^{t} x_{1}+2^{t} x_{0}+2^{m-1}\right) .
\end{aligned}
$$

Таким образом, $\quad \Delta_{+}^{(m)} g^{[2]}(x) \equiv{ }_{2^{n}} 2^{m-1}, \quad$ т.е. $\quad d_{+}\left(g^{[2]}\right)=m . \quad$ Если $t \in\{m+1, \ldots, 2 m\}$, то

$$
\begin{aligned}
& \psi_{2}^{-1}\left(\Delta_{+}^{(t)} g^{[2]}(x)\right) \equiv_{2^{m}} \\
& \equiv \sum_{2^{m}} \sum_{c_{2} \in Z_{2^{t-m}}} \sum_{c_{1} \in Z_{2^{m}}}\left(2\left(x_{1}+c_{1}\right)+x_{0}+2^{2 m-t} c_{2}, x_{1}+c_{1}+x_{0}+2^{2 m-t} c_{2}\right)= \\
& \equiv \sum_{2^{m}} \sum_{c_{2} \in Z_{2^{t-m}}}\left(2^{m+1} x_{1}+2^{m}+2^{m} x_{0}+2^{3 m-t} c_{2}, 2^{m} x_{1}+2^{m-1}+2^{m} x_{0}+2^{3 m-t} c_{2}\right) \equiv_{2^{m}}(0,0) .
\end{aligned}
$$

Лемма доказана.

Покажем, что и для других аналогичных отображений $\Delta_{+}$-степень орбитальной производной не превосходит $m$.

Лемма 3.5. Пусть $m \in \mathbb{N}, n=r \cdot m \geq 2, f_{0}^{(j)} \in P\left(\mathbb{Z}_{2^{m}}\right), f_{1}^{(j)}: \mathbb{Z}_{2^{m}}^{r-1} \rightarrow \mathbb{Z}_{2^{m}}$, $j=0, \ldots, r-1$, и отображение $g \in P\left(\mathbb{Z}_{2^{n}}\right)$ задано равенством

$$
\begin{array}{r}
g\left(x_{r-1}, \ldots, x_{0}\right) \equiv_{2^{m}}\left(f_{0}^{(r-1)}\left(x_{r-1}\right)+f_{1}^{(r-1)}\left(x_{r-2}, \ldots, x_{0}\right), \ldots\right. \\
\left.\ldots, f_{0}^{(0)}\left(x_{r-1}\right)+f_{1}^{(0)}\left(x_{r-2}, \ldots, x_{0}\right)\right) .
\end{array}
$$

Тогда $d_{+}\left(g^{[r]}\right) \leq m$.

Доказательство следует из сравнения $\psi_{r}^{-1}\left(\Delta_{+}^{(m)} g^{[r]}(x)\right) \equiv_{2^{m}}$ $\equiv_{2^{m}}\left(y^{(r-1)}, \ldots, y^{(0)}\right)$, где

$$
y^{(i)} \equiv \sum_{2^{m}} \sum_{\beta \in Z_{2^{m}}} f_{0}^{(i)}(\beta), \quad i=0, \ldots, r-1 .
$$

Рассмотрим множество $P^{(n, r)} \subset S\left(\mathbb{Z}_{2^{n}}\right)$ всех подстановок $s$, представимых в виде

$$
s\left(\alpha+2^{n-r} c\right) \equiv_{2^{n}} \alpha^{g_{c}}+2^{n-r} v_{\alpha, c}
$$


где $\quad \alpha \in Z_{2^{n-r}}, \quad g_{c} \in S\left(Z_{2^{n-r}}\right), \quad v_{\alpha, c} \in Z_{2^{r}}, \quad c=0, \ldots, 2^{r}-1 . \quad$ Пусть $\quad \lambda^{(n, r)}$ : $s \mapsto\left(g_{0}, \ldots, g_{2^{r}-1}\right)$. Отметим, что $\left|P^{(n, r)}\right|=\left(2^{n-r} !\right)^{2^{r}}\left(2^{r} !\right)^{2^{n-r}}$.

Если $r \in\{0, \ldots, n-1\}, t \in Z_{r}, j=0, \ldots, 2^{n-r+t}-1$ и $W^{(t)}=\left(W_{0}, \ldots, W_{2^{n-r+t}-1}\right)$, где $W_{j}=\left\{\beta \in \mathbb{Z}_{2^{n}} \mid \beta \equiv_{2^{n-r+t}} j\right\}$, то $P^{(n, r)} \cap I G_{W^{(t)}} \neq \varnothing$.

Зададим отображение $\bar{\Delta}_{+}^{(r)} s: Z_{2^{n-r}} \rightarrow \mathbb{Z}_{2^{n}}$ равенством $\bar{\Delta}_{+}^{(r)} s(\alpha)=\Delta_{+}^{(r)} s(\alpha)$ для всех $\alpha \in Z_{2^{n-r}}$, т. е. от области определения $\mathbb{Z}_{2^{n-r}}$ оператора $\Delta_{+}^{(r)}$ перешли к области определения $Z_{2^{n-r}}$ оператора $\bar{\Delta}_{+}^{(r)}$.

Лемма 3.6. Пусть $n \geq 2, \quad r \in\{1, \ldots, n-1\}, \quad s \in P^{(n, r)}, \quad \lambda^{(n, r)}(s)=$ $=\left(g_{0}, \ldots, g_{2^{r}-1}\right)$.

1. Отображение $u_{s}^{(r)}: \mathbb{Z}_{2^{n-r}} \rightarrow \mathbb{Z}_{2^{n-r}}$, заданное равенством $u_{s}^{(r)}(\alpha) \equiv{ }_{2^{n-r}}$ $\equiv_{2^{n-r}} \Delta_{+}^{(r)} s(\alpha)$, не биективно.

2. Пусть $s\left(\alpha+2^{n-r} c\right) \equiv{ }_{2^{n}} \alpha^{g_{c}}+2^{n-r} v_{\alpha, c}, \quad\left\{v_{\beta, c} \mid c \in Z_{2^{r}}\right\}=Z_{2^{r}}$ для каждого $\beta \in Z_{2^{n}}$. Отображение $\bar{\Delta}_{+}^{(r)} s: Z_{2^{n-r}} \rightarrow \mathbb{Z}_{2^{n}}$ тогда и только тогда инъективно, когда инъективно отображение $f: Z_{2^{n-r}} \rightarrow \mathbb{Z}_{2^{n}}$, где $f(x) \equiv{ }_{2^{n}} g_{0}(x)+\ldots+g_{2^{r}-1}(x)$.

Доказательство. 1. Для произвольного $\alpha \in Z_{2^{n}}$ справедливо сравнение

$$
\Delta_{+}^{(r)} s(\alpha) \equiv{ }_{2^{n}} g_{0}(\alpha)+\ldots+g_{2^{r}-1}(\alpha)+2^{n-r} c
$$

где $c \in Z_{2^{r}}$. Значит,

$$
\begin{gathered}
\Delta_{+}^{(r)} s(\alpha) \equiv{ }_{2^{n-r}} g_{0}(\alpha)+\ldots+g_{2^{r}-1}(\alpha) . \\
\text { Отметим, что } \sum_{\beta \in Z_{2^{t}}} h(\beta) \equiv_{2^{t}} \sum_{\beta \in Z_{2^{t}}} \beta \equiv 2^{t-1} \text { для произвольных числа } t \in \mathbb{N}
\end{gathered}
$$
и подстановки $h \in S\left(\mathbb{Z}_{2^{t}}\right)$. Поэтому

$$
\sum_{\alpha \in Z_{2^{n-r}}} \Delta_{+}^{(r)} s(\alpha) \equiv_{2^{n-r}} \sum_{\alpha \in Z_{2^{n-r}}} g_{0}(\alpha)+\ldots+\sum_{\alpha \in Z_{2^{n-r}}} g_{2^{r}-1}(\alpha) \equiv_{2^{n-r}} 2^{n-r-1} \cdot 2^{r} \equiv_{2^{n-r}} 0 .
$$

Таким образом, отображение $u_{s}^{(r)}$ не биективно на $Z_{2^{n-r}}$. 
2. Для каждого $\alpha \in Z_{2^{r}}$ справедливы сравнения

$$
\begin{aligned}
& \Delta_{+}^{(r)} s(\alpha) \equiv{ }_{2^{n}} g_{0}(\alpha)+\ldots+g_{2^{r}-1}(\alpha)+\sum_{c \in Z_{2^{r}}} v_{\alpha, c} \equiv \equiv_{2^{n}} \\
& \equiv_{2^{n}} g_{0}(\alpha)+\ldots+g_{2^{r}-1}(\alpha)+2^{r-1}\left(2^{r}-1\right) .
\end{aligned}
$$

Таким образом, для каждого $\alpha \in Z_{2^{n-r}}$ имеем

$$
\Delta_{+}^{(r)} s(\alpha) \equiv_{2^{n}} \bar{\Delta}_{+}^{(r)} s(\alpha) \equiv_{2^{n}} f(\alpha)+2^{r-1}\left(2^{r}-1\right) .
$$

Значит, $\bar{\Delta}_{+}^{(r)} s$ инъективно, когда инъективно отображение $f$. Лемма доказана.

Приведем примеры инъективных отображений $\bar{\Delta}_{+}^{(r)} s$, где $s \in P^{(n, r)}$.

Примеры. Пусть $s \in P^{(n, r)}, \lambda^{(n, r)}(s)=\left(g_{0}, \ldots, g_{2^{r}-1}\right)$. Рассмотрим следующие два класса отображений кольца $\mathbb{Z}_{2^{n}}$.

1. Пусть $g_{i}(\alpha) \equiv_{2^{n-r}} a_{i} g_{0}(\alpha)+b_{i}$ для всех $\alpha \in Z_{2^{n-r}}$, где $a_{i}, b_{i} \in Z_{2^{n-r}}$, $\sum_{i=0}^{2^{r}-1} a_{i} \#_{2^{r+1}} 0, i=0, \ldots, 2^{r}-1$. Если $\left\{v_{\beta, c} \mid c \in Z_{2^{r}}\right\}=Z_{2^{r}}$ для каждого $\beta \in Z_{2^{n}}$, то инъективно отображение $f: Z_{2^{n-r}} \rightarrow \mathbb{Z}_{2^{n}}$, где $f(x) \equiv_{2^{n}} g_{0}(x)+\ldots+g_{2^{r}-1}(x)$. Из п. 2 леммы 3.6 следует, что отображение $\bar{\Delta}_{+}^{(r)} s: Z_{2^{n-r}} \rightarrow \mathbb{Z}_{2^{n}}$ инъективно.

2. Пусть $n=2 r,\left\{g_{0}(\delta), \ldots, g_{2^{r}-1}(\delta)\right\}=Z_{2^{r}}$ для каждого $\delta \in Z_{2^{r}}$. Тогда для любых элементов $\alpha, \alpha^{\prime} \in Z_{2^{\prime}}$ существует такой элемент $c_{\alpha, \alpha^{\prime}} \in \mathbb{Z}$, что

$$
\Delta_{+}^{(r)} s\left(\alpha^{\prime}\right)-\Delta_{+}^{(r)} s(\alpha) \equiv_{2^{n}} c_{\alpha, \alpha^{\prime}} 2^{r} .
$$

Кроме того, если

$$
\left\{v_{\beta, c} \mid c \in Z_{2^{r}}\right\}=Z_{2^{r}} \text { для каждого } \beta \in Z_{2^{n}},
$$

то из соотношения (3.2) следует, что для каждого $\alpha \in Z_{2^{r}}$ справедливы сравнения

$$
\Delta_{+}^{(r)} s(\alpha) \equiv_{2^{n}} \bar{\Delta}_{+}^{(r)} s(\alpha) \equiv_{2^{n}} 2^{r}\left(2^{r}-1\right)
$$

\section{4. Структурные свойства орбитальных производных}

Ряд криптографических свойств двоичных отображений векторных пространств формулируется в терминах сбалансированности производных. 
Очевидно, что при фиксированных $\alpha \in \mathbb{Z}_{2^{n}}, t \in\{1, \ldots, n-1\}$ орбитальная производная $\Delta_{+}^{(t)} s(\alpha)$ сбалансирована при случайном и равновероятном выборе отображения $s$ из $P\left(\mathbb{Z}_{2^{n}}\right)$, т. е. $\mathbf{P}\left\{\Delta_{+}^{(t)} s(\alpha) \equiv{ }_{2^{n}} \beta\right\}=2^{-n}$ для любого $\beta \in Z_{2^{n}}$. Покажем, что орбитальная производная не является сбалансированной при случайном и равновероятном выборе подстановки $s$ из $S\left(\mathbb{Z}_{2^{n}}\right)$.

Заметим, что для подстановки $s$ множества значений отображений $\Delta_{\oplus, \beta} s, \Delta_{+}^{(1)} s$ могут различаться при $\beta \in V_{n}^{\times}$. Так, $\Delta_{\oplus, \beta} g(\alpha) \neq 0$ для всех $g \in S\left(V_{n}\right), \quad \beta \in V_{n}^{\times}, \quad \alpha \in V_{n}$. Однако $\Delta_{+}^{(1)} g^{\prime}(\alpha) \equiv_{2^{n}} 0$ для всех таких пар $\left(g^{\prime}, \alpha\right) \in S\left(\mathbb{Z}_{2^{n}}\right) \times \mathbb{Z}_{2^{n}}$, что $g^{\prime}(\alpha) \notin\left\{0,2^{n-1}\right\}, g^{\prime}\left(\alpha+2^{n-1}\right)=2^{n}-g^{\prime}(\alpha)$.

Утверждение 4.1. Пусть $n \geq 2, \alpha \in \mathbb{Z}_{2^{n}}, s \in \in_{U} S\left(\mathbb{Z}_{2^{n}}\right), \beta \in Z_{2^{n}}$. Тогда

$$
\mathbf{P}\left\{\Delta_{+}^{(1)} s(\alpha) \equiv_{2^{n}} \beta\right\}= \begin{cases}1 /\left(2^{n}-1\right), & \text { если } \beta \equiv_{2} 1, \\ 1 / 2^{n}-1 /\left(2^{2 n}-2^{n}\right), & \text { если } \beta \equiv_{2} 0 .\end{cases}
$$

Доказательство следует из равенств

$$
\begin{aligned}
& \mathbf{P}\left\{\Delta_{+}^{(1)} s(\alpha) \equiv_{2^{n}} \beta\right\}=\sum_{\delta \in X_{\beta}} \mathbf{P}\left\{s(\alpha)=\delta, s\left(\alpha+2^{n-1}\right) \equiv_{2^{n}} 2^{n}+\beta-\delta\right\}= \\
& = \begin{cases}2^{n} /\left(2^{2 n}-2^{n}\right), & \text { если } \beta \equiv_{2} 1, \\
\left(2^{n}-2\right) /\left(2^{2 n}-2^{n}\right), & \text { если } \beta \equiv_{2} 0,\end{cases}
\end{aligned}
$$

где

$$
X_{\beta}= \begin{cases}Z_{2^{n}}, & \text { если } \beta \equiv_{2} 1, \\ Z_{2^{n}} \backslash\left\{\beta / 2, \beta / 2+2^{n-1}\right\}, & \text { если } \beta \equiv_{2} 0 .\end{cases}
$$

Для отображения $g \in P\left(\mathbb{Z}_{2^{n}}\right)$ положим

$$
\vec{\Delta}_{+}^{(t)} g=\left(\Delta_{+}^{(t)} g(0), \ldots, \Delta_{+}^{(t)} g\left(2^{n}-1\right)\right) .
$$

Так как $\Delta_{+}^{(t)} g(i) \equiv_{2^{n}} \Delta_{+}^{(t)} g\left(i^{\prime}\right)$ при $i \equiv_{2^{n-t}} i^{\prime}, i, i^{\prime} \in \mathbb{Z}_{2^{n}}$, то достаточно рассматривать только первые $2^{n-t}$ координат вектора $\vec{\Delta}_{+}^{(t)} g$. Обозначим

$$
\tilde{\Delta}_{+}^{(t)} g=\left(\Delta_{+}^{(t)} g(0), \ldots, \Delta_{+}^{(t)} g\left(2^{n-t}-1\right)\right), t \in\{1, \ldots, n\} .
$$


Для $t \in\{1, \ldots, n-1\}$ положим $\tilde{\Delta}_{+}^{(t, n)} S=\left\{\tilde{\Delta}_{+}^{(t)} s \mid s \in S\left(\mathbb{Z}_{2^{n}}\right)\right\}$. Отметим, что $\left|\tilde{\Delta}_{+}^{(1, n)} S\right|>\left|\tilde{\Delta}_{+}^{(2, n)} S\right|>\ldots>\left|\tilde{\Delta}_{+}^{(n, n)} S\right|, \quad \tilde{\Delta}_{+}^{(n, n)} S=\left\{\left(2^{n-1}\right)\right\}, \quad$ так $\quad$ как $\Delta_{+}^{(n)} s(0) \equiv_{2^{n}} \sum_{i=0}^{2^{n}-1} i \equiv_{2^{n}} 2^{n-1}$ для каждой подстановки $s \in S\left(\mathbb{Z}_{2^{n}}\right)$. Кроме того, если $\beta=\left(\beta_{0}, \ldots, \beta_{2^{n-t}-1}\right) \in Z_{2^{n}}^{2^{n-t}}$ и $\beta \notin \tilde{\Delta}_{+}^{(t, n)} S$, то $\beta$ - невозможный вектор, т. е. для каждой подстановки $s \in S\left(\mathbb{Z}_{2^{n}}\right)$ не выполняются сравнения

$$
\sum_{\delta \in U_{n, n-r}(i)} \delta^{s} \equiv \beta_{2^{n}} \beta_{i}, i=0, \ldots, 2^{n-r}-1
$$

Для $m<n$ укажем некоторые соответствия между множествами значений орбитальных производных $\tilde{\Delta}_{+}^{(t, m)} S$ и $\tilde{\Delta}_{+}^{(t, n)} S$. В частности, приведем подстановки $s \in S\left(\mathbb{Z}_{2^{n}}\right)$ со свойством $\tilde{\Delta}_{+}^{(t)} s \in_{2^{m}} \tilde{\Delta}_{+}^{(t, m)} S$. Тем самым будет показано, что любому набору из $\tilde{\Delta}_{+}^{(t, m)} S$ соответствует некоторое подмножество из $\tilde{\Delta}_{+}^{(t, n)} S$.

Утверждение 4.2. Пусть $n \geq 2, \quad m, n, t \in \mathbb{N}, \quad t \leq m<n$, $\left(\beta_{j \cdot 2^{m-t}}, \ldots, \beta_{(j+1) \cdot 2^{m-t}-1}\right) \in \tilde{\Delta}_{+}^{(t, m)} S$ для $j=0, \ldots, 2^{n-m}-1, \quad \beta=\left(\beta_{0}, \ldots, \beta_{2^{n-t}-1}\right), h \in S\left(\mathbb{Z}_{2^{n-t}}\right)$, $s \in_{U} S\left(\mathbb{Z}_{2^{n}}\right)$. Тогда

$$
\mathbf{P}\left\{\Delta_{+}^{(t)} s(i) \equiv_{2^{m}} \beta_{h(i)}, i=0, \ldots, 2^{n-t}-1\right\} \geq\left(2^{n-m} !\right)^{2^{m}}\left(2^{t} !\right)^{2^{n-t}} / 2^{n} !
$$

Доказательство. Пусть $\mathrm{B}_{m}^{(t, j)}=\left(B_{j 2^{m-t}}, \ldots, B_{(j+1) 2^{m-t}-1}\right)$ - такое разбиение множества $Z_{2^{m}}$, что $\sum_{\alpha \in B_{j 2^{m-t}+c}} \alpha \equiv_{2^{m}} \beta_{j \cdot 2^{m-t}+c}, c=0, \ldots, 2^{m-t}-1, j=0, \ldots, 2^{n-m}-1$.

Рассмотрим отображения $\sigma_{i} \in S\left(\mathbb{Z}_{2^{n-m}}\right), i=0, \ldots, 2^{m}-1$ и множества $B_{j 2^{m-t}+c}^{\prime}=\left\{\alpha+2^{m} \sigma_{p}(j) \mid p \in B_{j 2^{m-t}+c}\right\}, \quad c=0, \ldots, 2^{m-t}-1, \quad j=0, \ldots, 2^{n-m}-1$. Очевидно, что $B^{\prime}=\left(B_{0}^{\prime}, \ldots, B_{2^{n-1}-1}^{\prime}\right)-$ разбиение множества $Z_{2^{n}}$. Число всех таких разбиений множества $Z_{2^{n}}$, определяемых разбиениями $\mathrm{B}_{m}^{(t, j)}$ для $j=0, \ldots, 2^{n-m}-1$, равно $d_{n, m}=\left(2^{n-m} !\right)^{2^{m}}$.

Рассмотрим множество подстановок

$$
G\left(B^{\prime}\right)=\left\{g \in S\left(\mathbb{Z}_{2^{n}}\right) \mid\left(U_{n, t}(i)\right)^{g}=B_{h(i)}^{\prime}, i=0, \ldots, 2^{n-t}-1\right\} .
$$


Учитывая, что $\left|G\left(B^{\prime}\right)\right|=\left(2^{t} !\right)^{2^{n-t}}$, имеем

$$
\mathbf{P}\left\{\Delta_{+}^{(t)} S(i) \equiv_{2^{m}} \beta_{h(i)}, i=0, \ldots, 2^{n-t}-1\right\} \geq\left|G\left(B^{\prime}\right)\right| d_{n, m} / 2^{n} !
$$

Утверждение доказано.

Покажем, что орбитальная производная позволяет разбивать ключевые множества на классы «эквивалентности». В частности, для ключей шифрования, принадлежащих одному классу, одинаковы значения орбитальной производной $r$-го порядка функции зашифрования на этих ключах. Данные множества ключей и их представители несложно описываются. Это может быть использовано при разработке метода криптоанализа типа «эквивалентных» ключей.

Пусть $l \in \mathbb{N}, k_{i}-i$-й раундовый ключ, $\left(k_{1}, \ldots, k_{l}\right) \in \mathbb{Z}_{2^{n}}^{l}, g_{k_{i}}^{(i)}$ - раундовая функция $i$-го раунда алгоритма блочного шифрования, $g_{k_{i}}^{(i)} \in S\left(Z_{2^{n}}\right)$, $g_{\left(k_{1}, \ldots, k_{l}\right)}=g_{k_{1}}^{(1)} \ldots g_{k_{l}}^{(l)}, \delta^{(0)} \in Z_{2^{n}}, \delta^{(l)}=\delta^{(0)} g_{\left(k_{1}, \ldots, k_{l}\right)}, B g_{\left(k_{1}, \ldots, k_{l}\right)}=\left\{\beta g_{\left(k_{1}, \ldots, k_{l}\right)} \mid \beta \in B\right\}$, $B \subseteq Z_{2^{n}}$.

В следующем утверждении операция «+» в индексах означает сложение по модулю $2^{n}$, если не оговорено противное.

Утверждение 4.3. Пусть $n \geq 2, \quad l \in \mathbb{N}, t \in\{1, \ldots, n-1\},\left(k_{1}, \ldots, k_{l}\right) \in \mathbb{Z}_{2^{n}}^{l}$, $h^{(i)} \in S\left(\mathbb{Z}_{2^{n}}\right), \quad g_{k_{i}}^{(i)}: \lambda \mapsto\left(\lambda+k_{i}\right)^{h^{(i)}}, \quad i=1, \ldots, l, \quad \vec{\Delta}_{+}^{(t)} g_{\left(k_{1}, k_{2}, \ldots, k_{l}\right)}=\left(\alpha_{0}, \ldots, \alpha_{2^{n}-1}\right)$, $\beta, \beta^{\prime} \in \mathbb{Z}_{2^{n}}$. Тогда:

1) если $\beta \equiv_{2^{n-t}} \beta^{\prime}$, mо $\vec{\Delta}_{+}^{(t)} g_{\left(k_{1}+\beta, k_{2}, \ldots, k_{l}\right)} \equiv_{2^{n}} \vec{\Delta}_{+}^{(t)} g_{\left(k_{1}+\beta^{\prime}, k_{2}, \ldots, k_{l}\right)}$;

2) если $\vec{\Delta}_{+}^{(t)} g_{\left(k_{1}+\beta, k_{2}, \ldots, k_{l}\right)}=\left(\alpha_{0}^{\prime}, \ldots, \alpha_{2^{n}-1}^{\prime}\right)$, то $\alpha_{(i+\beta)_{(n-t)}}=\alpha_{i_{(n-t)}}^{\prime}$ для любых $i, \beta \in \mathbb{Z}_{2^{n}}$

3) если $d \in\{1, \ldots, l-1\}$, то

$$
\Delta_{+}^{(t)} g_{\left(k_{1}, k_{2}, \ldots, k_{d}\right)}(\lambda)+2^{t} k_{d+1} \equiv \sum_{2^{n}} \sum_{\beta \in U_{n, n-t}(\lambda)} \beta g_{\left(k_{1}, k_{2}, \ldots, k_{l-1}, k_{l}\right)}\left(g_{k_{l}}^{(l)}\right)^{-1} \ldots\left(g_{k_{d+2}}^{(d+2)}\right)^{-1}\left(h^{(d+1)}\right)^{-1},
$$

в частности,

$$
\Delta_{+}^{(t)} g_{\left(k_{1}, k_{2}, \ldots, k_{d}\right)}(\lambda) \equiv_{2^{t}} \sum_{\beta \in U_{n, n-t}(\lambda)} \beta g_{\left(k_{1}, k_{2}, \ldots, k_{l-1}, k_{l}\right)}\left(g_{k_{l}}^{(l)}\right)^{-1} \ldots\left(g_{k_{d+2}}^{(d+2)}\right)^{-1}\left(h^{(d+1)}\right)^{-1} .
$$

Доказательство. 1) Отметим, что $U_{n, n-t}(\lambda+\beta)=U_{n, n-t}\left(\lambda+\beta^{\prime}\right)$ для любого $\lambda \in \mathbb{Z}_{2^{n}}$ тогда и только тогда, когда $\beta \equiv_{2^{n-t}} \beta^{\prime}$. Значит, если $\beta \equiv_{2^{n-t}} \beta^{\prime}$, 
To

$$
\begin{aligned}
& \vec{\Delta}_{+}^{(t)} g_{\left(k_{1}+\beta, k_{2}, \ldots, k_{l}\right)}(\lambda) \equiv \sum_{2^{n}} \sum_{\theta \in U_{n, n-t}(\lambda)}(\theta) g_{k_{1}+\beta}^{(1)} \ldots g_{k_{l}}^{(l)}= \\
& \equiv \sum_{2^{n}} \sum_{\theta \in U_{n, n-t}(\lambda)}(\theta+\beta) g_{k_{1}}^{(1)} g_{k_{2}}^{(2)} \ldots g_{k_{l}}^{(l)} \equiv \sum_{2^{n}} \sum_{\theta \in U_{n, n-t}(\theta+\beta)}(\theta) g_{k_{1}}^{(1)} g_{k_{2}}^{(2)} \ldots g_{k_{l}}^{(l)} \equiv_{2^{n}} \\
& \equiv \sum_{2^{n}} \sum_{\theta \in U_{n, n-t}\left(\lambda+\beta^{\prime}\right)}(\theta) g_{k_{1}}^{(1)} g_{k_{2}}^{(2)} \ldots g_{k_{l}}^{(l)} \equiv{\overrightarrow{\Delta^{n}}}_{+}^{(t)} g_{\left(k_{1}+\beta^{\prime}, k_{2}, \ldots, k_{l}\right)}(\lambda) .
\end{aligned}
$$

2) Так как для любого $\lambda \in \mathbb{Z}_{2^{n}}$ выполняется сравнение

$$
\begin{aligned}
& \vec{\Delta}_{+}^{(t)} g_{\left(k_{1}+\beta, k_{2}, \ldots, k_{l}\right)}(\lambda) \equiv_{2^{n}} \sum_{\theta \in U_{n, n-t}(\lambda)}(\lambda+\beta) g_{k_{1}}^{(1)} g_{k_{2}}^{(2)} \ldots g_{k_{l}}^{(l)} \equiv \sum_{2^{n}} \\
& \equiv_{2^{n}} \sum_{\theta \in U_{n, n-t}(\lambda+\beta)}(\lambda) g_{k_{1}}^{(1)} g_{k_{2}}^{(2)} \ldots g_{k_{l}}^{(l)} \equiv \vec{\Delta}_{2^{n}}^{(t)} g_{\left(k_{1}, k_{2}, \ldots, k_{l}\right)}(\lambda+\beta),
\end{aligned}
$$

то $\alpha_{(i+\beta)_{(n-t)}} \equiv{ }_{2^{n}} \alpha_{i_{(n-t)}}^{\prime}, i=0, \ldots, 2^{n}-1$.

3) П.3 следует из сравнения

$$
\begin{gathered}
\sum_{\beta \in U_{n, n-t}(\lambda)} \beta g_{\left(k_{1}, k_{2}, \ldots, k_{l-1}, k_{l}\right)}\left(g_{k_{l}}^{(l)}\right)^{-1} \ldots\left(g_{k_{d+2}}^{(d+2)}\right)^{-1}\left(h^{(d+1)}\right)^{-1} \equiv_{2^{n}} \\
\equiv \sum_{2^{n}} \sum_{\beta \in U_{n, n-t}(\lambda)}\left(\beta g_{\left(k_{1}, k_{2}, \ldots, k_{d-1}, k_{d}\right)}+k_{d+1}\right) \equiv_{2^{n}} \\
\equiv 2^{t} k_{d+1}+\sum_{\beta \in U_{n, n-t}(\lambda)} \beta g_{\left(k_{1}, k_{2}, \ldots, k_{d-1}, k_{d}\right)} \equiv_{2^{n}} \Delta_{+}^{(t)} g_{\left(k_{1}, k_{2}, \ldots, k_{d}\right)}(\lambda)+2^{t} k_{d+1} .
\end{gathered}
$$

Утверждение доказано.

Отметим, что п.1 утверждения 4.3 дает пример ключей, на которых равны все значения орбитальной производной $t$-го порядка от функции зашифрования. Относительно нее их можно считать «эквивалентными», т. е. ключи $k=\left(k_{1}, \ldots, k_{l}\right), \quad k^{\prime}=\left(k_{1}^{\prime}, \ldots, k_{l}^{\prime}\right)$ считаются эквивалентными, если $k_{1} \equiv_{2^{n-t}} k_{1}^{\prime}$ и $k_{i}^{\prime}=k_{i}, i=2, \ldots, l$.

П. 2 дает пример «связанных» ключей. Он позволяет для ключа $\left(k_{1}, \ldots, k_{l}\right)$ и «связанного» ключа $\left(k_{1}^{\prime}, k_{2}, \ldots, k_{l}\right)$, где $k_{1}^{\prime} \equiv_{2^{n}} k_{1}+\beta$, определить зависимость между шифртекстами, полученными при зашифровании на соответствующих ключах.

Использование п. 3 утверждения 4.3 позволяет с помощью орбитальной производной $l$-раундовой функции зашифрования получать информацию об орбитальной производной $(l-1)$-раундовой функции зашифрования без знания раундового ключа $l$-го раунда. В частности, если ключевое мно- 
жество есть $\mathbb{Z}_{2^{n}}^{l}$, то трудоемкость метода полного перебора при использовании $t$-й орбитальной производной имеет порядок $O\left(2^{n(l-1)+t}\right)$ операций зашифрования.

Кроме того, соотношение (4.1) не зависит от раундового ключа $k_{d+1}$. Раундовые ключи $\left(k_{1}, \ldots, k_{d}\right),\left(k_{d+2}, \ldots, k_{l}\right)$ можно находить, применяя метод встречи посередине и равенство (4.1).

Утверждение 4.3 несложно переформулировать и для оператора $\Delta_{+, a}^{(t)}$, порожденного орбитами группы $\langle a\rangle, a \in \mathbb{Z}_{2^{n}}$, в группе $\mathbb{Z}_{2^{n}}^{+}$. Если $U_{n}^{(a)}(\alpha)=\alpha^{\langle a\rangle}$ - орбита элемента $\alpha$ в группе $\langle a\rangle$ и

$$
\Delta_{+, a} s(\alpha) \equiv_{2^{n}} \sum_{\beta \in U_{n}^{(a)}(\alpha)} s(\beta),
$$

Tо

$$
\begin{aligned}
& \Delta_{+, a} g_{\left(k_{1}, k_{2}, \ldots, k_{d}\right)}(\alpha)+\left|U_{n}^{(a)}(\alpha)\right| \cdot k_{d+1} \equiv_{2^{n}} \\
& \equiv_{2^{n}} \sum_{\beta \in U_{n}^{(a)}(\alpha)} \beta g_{\left(k_{1}, k_{2}, \ldots, k_{l-1}, k_{l}\right)}\left(g_{k_{l}}^{(l)}\right)^{-1} \ldots\left(g_{k_{d+2}}^{(d+2)}\right)^{-1}\left(h^{(d+1)}\right)^{-1} .
\end{aligned}
$$

Для $f \in P\left(V_{n}\right), W<V_{n}$ рассмотрим аналог утверждения 4.3 на векторном пространстве $V_{n}$ для оператора

$$
\Delta_{W, \oplus} f(\alpha)=\bigoplus_{\gamma \in W} f(\alpha \oplus \gamma) .
$$

Положим

$$
\vec{\Delta}_{W, \oplus} f=\left(\Delta_{W, \oplus} f(0), \ldots, \Delta_{W, \oplus} f\left(2^{n}-1\right)\right) .
$$

Утверждение 4.4. Пусть $n \geq 2, \quad l \in \mathbb{N}, \quad W<V_{n}, \quad\left(k_{1}, \ldots, k_{l}\right) \in V_{n}^{l}$, $h^{(i)} \in S\left(V_{n}\right), \quad g_{k_{i}}^{(i)}: \lambda \mapsto\left(\lambda \oplus k_{i}\right)^{h^{(i)}}, \quad i=1, \ldots, l, \quad \vec{\Delta}_{W, \oplus} g_{\left(k_{1}, k_{2}, \ldots, k_{l}\right)}=\left(\alpha_{0}, \ldots, \alpha_{2^{n}-1}\right)$, $\beta, \beta^{\prime} \in V_{n}$. Тогда:

1) если $\beta \oplus \beta^{\prime} \in W$, то $\vec{\Delta}_{W, \oplus} g_{\left(k_{1} \oplus \beta, k_{2}, \ldots, k_{l}\right)}=\vec{\Delta}_{W, \oplus} g_{\left(k_{1} \oplus \beta^{\prime}, k_{2}, \ldots, k_{l}\right)}$;

2) если $\vec{\Delta}_{W, \oplus} g_{\left(k_{1} \oplus \beta, k_{2}, \ldots, k_{l}\right)}=\left(\alpha_{0}^{\prime}, \ldots, \alpha_{2^{n}-1}^{\prime}\right)$, то $\alpha_{i \oplus \beta}=\alpha_{i}^{\prime}$ для любого $i \in Z_{2^{n}}$;

3) справедливо равенство

$$
\Delta_{W, \oplus} g_{\left(k_{1}, k_{2}, \ldots, k_{d}\right)}(\lambda)=\bigoplus_{\beta \in \lambda \oplus W} \beta g_{\left(k_{1}, k_{2}, \ldots, k_{l-1}, k_{l}\right)}\left(g_{k_{l}}^{(l)}\right)^{-1} \ldots\left(g_{k_{d+2}}^{(d+2)}\right)^{-1}\left(h^{(d+1)}\right)^{-1} .
$$

Доказательство аналогично доказательству утверждения 4.3. 
Для алгоритмов шифрования Фейстеля аналог утверждения 4.3 можно представить в следующем виде.

Утверждение 4.5. Пусть $l \in \mathbb{N}, \quad n=2 m \geq 4, \quad\left(k_{1}, \ldots, k_{l}\right) \in V_{m}^{l}$, $h^{(i)} \in S\left(V_{m}\right), g_{k_{i}}^{(i)}: V_{m}^{2} \rightarrow V_{m}^{2}$,

$$
g_{k_{i}}^{(i)}:\left(\lambda_{1}, \lambda_{0}\right) \mapsto\left(\lambda_{0}, \lambda_{1} \oplus\left(k_{i} \oplus \lambda_{0}\right)^{h^{(i)}}\right), i=1, \ldots, l,
$$

$\vec{\Delta}_{+}^{(t)} g_{\left(k_{1}, k_{2}, \ldots, k_{l}\right)}=\left(\alpha_{0}, \ldots, \alpha_{2^{n}-1}\right)$ для $t \in\{1, \ldots, n-1\}, \beta, \beta^{\prime} \in V_{m}, W<V_{m}$. Тогда:

1) если $\beta \oplus \beta^{\prime} \in W$, то

$$
\vec{\Delta}_{\{\tilde{0}\} \times W, \oplus} g_{\left(k_{1} \oplus \beta, k_{2}, \ldots, k_{l}\right)}=\vec{\Delta}_{\{\tilde{0}\} \times W, \oplus} g_{\left(k_{1} \oplus \beta^{\prime}, k_{2}, \ldots, k_{l}\right)} ;
$$

2) если $\vec{\Delta}_{W, \oplus} g_{\left(k_{1} \oplus \beta, k_{2}, \ldots, k_{l}\right)}=\left(\alpha_{0}^{\prime}, \ldots, \alpha_{2^{n}-1}^{\prime}\right)$, то $\alpha_{i \oplus(0, \beta)}=\alpha_{i}^{\prime}$ для любого $i \in Z_{2^{n}}$;

3) если $\delta \in V_{n}^{\times}, \lambda^{(0)} \in V_{n}, d \in\{0, \ldots, l-1\}$,

$$
\left(\lambda_{1}^{(j)}, \lambda_{0}^{(j)}\right)=\lambda^{(0)} g_{\left(k_{1}, \ldots, k_{j}\right)},\left(\lambda_{1}^{(j)}, \lambda_{0}^{\prime(j)}\right)=\left(\lambda^{(0)} \oplus \delta\right) g_{\left(k_{1}, \ldots, k_{j}\right)}, j=1, \ldots, l,
$$

mo

$$
\left(\lambda_{0}^{(d+1)} \oplus \lambda_{1}^{(d)}\right)^{\left(h^{(d+1)}\right)^{-1}} \oplus\left(\lambda_{0}^{\prime(d+1)} \oplus \lambda_{1}^{\prime(d)}\right)^{\left(h^{(d+1)}\right)^{-1}}=\lambda_{0}^{(d)} \oplus \lambda_{0}^{(d)} .
$$

Доказательство очевидно.

Как видно из утверждения 4.5, для алгоритма шифрования Фейстеля не удается получить аналог п.3 утверждения 4.3 , а имеет место более сложная зависимость между подблоками промежуточного текста $\lambda_{0}^{(d)}, \lambda_{0}^{(d)}$, и $\lambda_{0}^{(d+1)}, \lambda_{0}^{(d+1)}, d \in\{0, \ldots, l-1\}$. Она не позволяет в общем случае применять метод встречи посередине без учета одного раундового ключа, как это можно делать, используя п.3 утверждений 4.3 и 4.4. Таким образом, алгоритм шифрования Фейстеля является более стойким относительно подхода, основанного на методе встречи посередине и орбитальной производной, чем XSL-алгоритмы блочного шифрования.

\section{Список литературы}

1. Lai X. Higher order derivatives and differential cryptanalysis // Proc. Symp. on Communication, Coding and Cryptography. - 1994.- P. 227-233.

2. Марков А. А. Исчисление конечных разностей, 2 изд. - Одесса: 1910.

3. Березин И. С., Жидков Н. П. Методы вычислений. Т. 1-2, 3 изд. - М.: Наука, 1966. 
4. Гельфонд А. О. Исчисление конечных разностей. - М.: Наука, 1967.

5. Сачков В.Н. Введение в комбинаторные методы дискретной математики. - М.: МЦНМО, 2004.

6. Jaworski J., Tyksinski T. Bounds for differential probabilities in even order Abelian groups // Tatra Mount. Math. Publ. - 2008. - V. 4. - P. 33-46.

7. Погорелов Б.А., Пудовкина М.А. Орбитальные производные над кольцом вычетов. Часть І. Общие свойства // Математические вопросы криптографии. - 2014. - Т. 5. Вып. 4. - С. 99-127.

8. Погорелов Б.А., Пудовкина М.А. Факторструктуры преобразований // Математические вопросы криптографии. - 2002. - Т. 3. Вып. 3.- С. 81104.

9. Черемушкин A.B. Аддитивный подход к определению степени нелинейности дискретной функции // Прикладная дискретная математика. 2010. - Т. 8. Вып. 2. - С. 22-33. 